\title{
Utility of the computer as tachistoscope in a particular psychophysical setting*
}

\section{JOSEPH J. MEZRICH \\ Lniversity of Michigan, Ann Arbor, Michigan 48104}

A discussion is provided covering the use of the computer as a tachistoscope.

There is very little question that the introduction of the computer into the psychology laboratory has enriched the experimental repertoire of the psychologist. The impact of the laboratory computer has been quite evident in the field of visual perception. The use of dynamic visual noise by Uttal (e.g., 1969), the use of random-dot cinematograms by Julesz (e.g., 1971), and the study of stereotyped scanpaths for eye movements by Noton and Stark (1971) are but a few of the approaches to the analysis of visual processing that would be impossible without the use of the computer.

However, the vast majority of experiments being performed on the processing of information by the visual system do not require exotic stimulus presentation or data collection schemes. It is reasonable to ask what advantage is gained by employing a computer in such experiments, where more traditional apparatus is clearly adequate. Some insight concerning this question can be gained by examining the impact of on-line use of the computer on the procedures of a specific, traditional type of experiment as the degree of involvement of the computer increases. In particular, we will consider the studies of Reicher (1968). Wheeler (1970), and Mezrich (1973) on the word superiority effect.

In 1968, Reicher demonstrated that a four-letter word is more easily recognized than a single letter when both are briefly displayed for the same duration. For example, a $S$ presented with a brief visual display of the stimulus WORD and then asked to decide whether the letter $\mathrm{D}$ or the letter $\mathrm{K}$ was present in the stimulus is more likely to be correct than if the stimulus $\mathrm{D}$ preceded these response choices. It should be noted that either $\mathrm{D}$ or $\mathrm{K}$ can be used to construct a four-letter word with the initial letters WOR, so the possibility of response bias is absent. The phenomenon does not depend on the critical letter position of the word. Reicher's finding is generally referred to as the word superiority effect.

Some details about Reicher's experimental method are necessary for our discussion. Each stimulus trial was composed of a sequence of three visual displays: a prestimulus blank field with a fixation point. followed by the stimulus. which was followed by the two

\footnotetext{
*The valuable advice and assistance of J. E. Keith Smith and Frank M. Goode are gratefully acknowledged. The work reported in this paper was supported by Public Health Service Grant GM-01231-08 to the University of Michigan.
}

response alternatives located adjacent to a visual noise mask superimposed on the stimulus location. The noise mask was identical for every trial and consisted of a matrix of superimposed $\mathrm{Xs}$ and Os. The fact that this noise mask is far from random turns out to be unimportant. Reicher typed his presentation materials on white cards and used a Scientific Prototype Model GB three-channel tachistoscope as his display device.

An important part of the experimental procedure is the determination of the appropriate stimulus exposure duration for each S. For a two-alternative forced-choice situation, a stimulus exposure duration that insures response accuracy at some level between $50 \%$ and $100 \%$ correct is necessary. Reicher used what he called a modified up-down threshold procedure in a preliminary session with each $S$ to ascertain the appropriate exposure duration. One hundred and twenty stimuli were presented in blocks of 10 . Based on performance for a given block, the exposure duration for the next block was adjusted up, down, or not at all. The exposure duration determined from the last block was used for the experimental sessions, which occurred on subsequent days. Reicher does not give full details about the scenario of this preliminary session, but it would appear to have taken at least $1 \mathrm{~h}$.

It should be clear that there is little justification for assuming that such a technique yields a reliable estimate of the appropriate stimulus exposure duration for a $S$ in subsequent sessions of the experiment. The problem of the instability of operating levels in psychophysical experiments is well known (e.g., Green \& Swets, 1966). The effects of fatigue, motivation, and practice within a session all contribute to, or detract from, the performance of the $S$. There is no guarantee that the state of the $\mathrm{S}$ is constant within a session, much less between sessions that occur on different days. Fortunately, the word superiority effect seems to occur at all accuracy levels in the $50 \%$ to $100 \%$ correct range, even though the magnitude of the effect does depend on the value of the accuracy level. However, the problem of operating-level fluctuations makes these results less convincing than they could be.

To a great extent, Reicher was forced to separate the preliminary session from the experimental sessions because a conventional tachistoscope was used. The somewhat clumsy procedure of having the $E$ continuously monitor a S's performance level and adjust the tachistoscope exposure setting practically insures a fairly long preliminary session. To precede a long experimental session with such a preliminary session would surely introduce a significant amount of fatigue 
and boredom, very likely making the estimate of the appropriate exposure duration as unreliable as if the two sessions were separated by days. Thus. the procedural constraints imposed by his equipment were quite severe.

Another aspect of Reicher's procedure that we would like to focus attention on is the postexperimental questioning of Ss. As is typical for experiments of this nature, the $S$ is called back for discussion at least a day after his participation in the experiment. This delay is used so that the $E$ can have the relevant performance indicators in hand to help guide his questioning. Maximum yield from discussions with Ss about factors they suspect may have affected performance occurs when the experiment is still very fresh in the S's mind, and when the $E$ has available performance indicators to guide the discussion. Because traditional data-collection procedures were used, Reicher had to compromise on the first of these conditions in order to exploit the second. Surely, a significant amount of detail is forgotten by the $S$ when he has to wait at least a day to report his experiences. It is probably obvious tiat on-line use of a computer enables one to insure presence of the conditions for maximum discussion yield. Further discussion on this point appears below.

Two years after Reicher found the word superiority effect, Wheeler (1970) performed a series of experiments to explore a number of hypotheses about sources of the effect. His conclusion was that the effect is quite real, but a simple explanation of its cause is elusive. Wheeler used a PDP-8 computer connected to an oscilloscope as a tachistoscope. The machine was also used for data collection and for some on-line computation.

A major difference between the visual displays used by Reicher and by Wheeler was the noise mask employed. It was pointed out above that the fact that Reicher did not use a random noise mask was unimportant. This is known primarily because Wheeler used a random noise mask at the offset of each stimulus and still got the effect. His mask consisted of a random pattern of dots, different on every trial. Such a mask is easily generated with a computer-controlled display, but is impractical with a traditional tachistoscope display. The use of a nonrandom display to mask patterned visual stimulation introduces potential artifacts, making Reicher's results less convincing than they could be. Thus, the accessibility of random noise masks illustrates one of the advantages of the computer as tachistoscope in this experimental setting.

We pointed out, above, the disadvantages of Reicher's procedure for determining the stimulus exposure duration. Wheeler apparently used a similar algorithm for determining this experimental parameter (precise details were not given in his paper); however, the advantage of computer control gives the algorithm a bit more reliability. For a typical session in Wheeler's experiment, which lasted about $1 \mathrm{~h}$, the $\mathrm{S}$ responded to 384 stimuli. The first half of these stimuli were used to determine the appropriate stimulus exposure duration for the second half, or the first half hour was spent as preliminary session for the second half hour. Because a computer was used, Wheeler was able to accomplish with a $S$ in an hour what it took Reicher two long sessions over a period of 2 days to do. The simple act of computerizing the data-collection procedure enabies the $S$ to run through the preliminary and experimental sessions at a fairly rapid pace, thereby shortenung the durations of both sessions. As the durations of the sessions decreasc, so does the problent of operating level instability. Thus, another advantage of the computer in this experiment becomes evident. It wiil be seen shorty that orie can make more efficient use of the computer for ininimizing the instability problem.

The last comment we would like to make about Whesler's method concerns his approach to postexperimental questioning of $\mathrm{S}$ s. Whereas Reicher postponed $S$ questioning until performance indicators wer: available, Wheeler engaged in immediate postexperimental interview. Unfortunately, he did not program the computer to print out performance levels prior to this interview, thus the conditions for maximum discussion yield were not met

We performed yet another set of experiments on the ritysterious word superiority effect (Mezrich, 1973). We confirmed the findings of Reicher and Wheeler, and also found that forcing Ss to vocalize all stimuli reverses the effect. making letters more recognizable than words. A CRT controlled by a LINC -8 computer was used as a tachistoscope. The computer was also used for data collection and on-line computation.

Wo used a dynamic random noise mask. ${ }^{1}$ A continuously changing display of lines of random length, orientation, and location was painted over the stimulus location at stimulus offset, giving the visual effect of sntali sticks flying about. The use of this type of random mask was partly due to convenience and partly for reasons of aesthetics. We were concerned about preventing Ss from becoming bored during our experiments. Making an otherwise dull display interesting to watch was intended as a boredom inhibitor. Clearly, a noise mask such as ours is impossible to generate with a conventional tachistoscope.

In constructing the actual stimulus display, the $\mathrm{E}$ using the computer as a tachistoscope has a few more things to worry about than does the $E$ using a traditional tachistoscope. In our displays, a letter was constructed from a grid of points rather than from continuous strokes. We, therefore, had to be sure that the distinctiveness and clarity of a briefly presented letter was not affected by its being spatially discrete. It was not. The stimulus exposure duration for a CRT display is necessarily quantized. since the duration of exposure is an integral multiple of the duration of a single painting of the stimulus. In our experiments, we avoided any difficulties due to quantization by using a very brief-duration painting cycle. Wheeler's solution to this problem was, apparently, to use a storage-scope in the store mode. Slow drifts in luminosity of the CRT display and a long time constant CRT phosphor are two 
equipment properties that the $\mathrm{E}$ has to guard against in selecting the oscilloscope that his computer will control. Adequate CRTs are readily available, so this is not a serious problem. Any disadvantages caused by using a CRT display in the type of experiment we are discussing are insignificant.

In reviewing the approaches of Reicher and Wheeler to the problem of determining the stimulus exposure duration, we pointed out that, because of instability of operating levels, it is important to have a quick determination of the appropriate exposure duration, followed by a quick run through the experiment. In our experiments, a very quick exposure-duration determination was achieved by using a Markov design procedure due to Smith (1961).

After a brief instructional period, a $\mathrm{S}$ was run through 96 stimulus trials at 60-msec stimulus exposure duration. This warm-up session, which lasted about $15 \mathrm{~min}$, was included to eliminate practice effects before the exposure duration for the experimental session was determined. After a $2 \cdot \mathrm{min}$ break, the $\mathrm{S}$ was run through what we call the timing phase of the experiment. The algorithm for this timing phase was based on the following considerations. Let $p_{i}$ be the probability of a correct detection for a given type of stimulus material at exposure duration $t_{i}$. Only single-letter stimuli were used for the timing phase, so $p_{i}$ represents the hit rate for letters. The probability of two consecutive hits at exposure duration $t_{i}$ is, therefore, $p_{i}^{2}$. If we find the exposure duration at which the probability of two consecutive hits equals the probability of not getting two consecutive hits, then we have found $t_{i}$ where $p_{i}^{2}=$ $1-p_{i}^{2}$, or $p_{i}=.707$. In our algorithm, we started at an arbitrary exposure duration, $60 \mathrm{msec}$, and inspected each consecutive pair of responses on-line. Let $\mathrm{H}$ denote a hit, and $\mathbf{M}$ denote a miss. The four possible outcomes for a pair of responses are: $(\mathrm{H}, \mathrm{H}),(\mathrm{H}, \mathrm{M}),(\mathrm{M}, \mathrm{H})$, and $(\mathrm{M}, \mathrm{M})$. If $(\mathrm{H}, \mathrm{H})$ occurred, the exposure duration was decreased by $10 \mathrm{msec}$ for the next pair of stimuli. If one of the other three outcomes occurred, the exposure duration was increased by $10 \mathrm{msec}$. Actually, the algorithm also inspected the first response of a pair, before the second trial was initiated, and automatically increased the exposure duration by $10 \mathrm{msec}$ if a miss occurred on that trial, since $(\mathrm{H}, \mathrm{H})$ was not possible for that pair. We used $10-\mathrm{msec}$ increments because of display constraints. This hunting procedure continued until the exposure duration hopped back and forth between two 10-msec separated exposure durations four times in a row. The program had then found two exposure durations, $t_{0}$ and $t_{0}+10 \mathrm{msec}$, such that about midway between them the probability of two consecutive hits equals the probability of not getting two consecutive hits, or equivalently, the probability of a hit is .707 . The exposure duration was set at $t_{0}+$ $10 \mathrm{msec}$ for the following experimental session.

Two important advantages are offered by this procedure. First, it gives a very quick exposure determination. It usually took less than 5 min for the stopping criterion to be reached. Second, it gives a more rigorous basis, on statistical grounds, than a modified up-down threshold procedure for assuming that we have found the exposure duration we want. This procedure reflects our philosophy about the optimal way of insuring desired operating levels. After practice effects are eliminated by a warm-up session, a very quick probe is used to set the $S$ at the operating level desired and then the experiment proper is run. On-line use of the computer makes the quick probe easy to implement.

We have pointed out the conditions that we feel are necessary for obtaining maximum yield from S's reports, i.e., immediate access to the S's insights and to the relevant performance indicators at the termination of the experimental session. In our experiments, the S's response to the last stimulus of the experiment brought in a subroutine that did the appropriate computations and printed out the desired performance indicators. This printout included a summary of accuracy levels and confidence-level judgments. By the time the $S$ got out of his chair, the E had a complete summary of the S's performance. Thus, the $S$ and $E$ could immediately discuss factors that may have affected performance, as indicated on the computer printout. In our pilot studies, this minimal-delay feedback was a critical input that led to the realization that vocalization might reverse the word superiority effect. The availability of minimal-delay feedback, for use in immediate postexperimental discussion with pilot $S s$, is an important asset for structuring an experiment. This asset is not available to Es who do not use a computer.

In examining the utility of the computer as tachistoscope in studies on the word superiority effect, we have pointed out a number of procedural constraints, imposed by the use of traditional apparatus in a traditional type of experiment, that are easily avoided by computerization. We have focused our attention on these studies of the word superiority effect in order to be concrete. The points we have considered, however, are really quite general and are applicable to a number of experimental settings.

\section{REFERENCES}

Green, D. M., \& Swets, J. A. Signal detection theory and psychophysics. New York: Wiley, 1966.

Julesz, B. Foundations of cyclopean perception. Chicago: University of Chicago Press, 1971.

Mezrich, J. J. The word superiority effect in brief visual displays. Elimination by vocalization. Perception \& Psychophysics, $1973,13,45-48$.

Noton, D., \& Stark, L. Scanpaths in saccadic eye movements while viewing and recognizing patterns. Vision Research, 1971, 11, 929-942.

Reicher, G. M. Perceptual recognition as a function of meaningfulness of stimulus material. Technical Report No. 7 , Human Performance Center, University of Michigan, 1968.

Smith, J. E. K. Stimulus programming in psychophysics. Psychometrika, 1961, 26, 27-33.

Uttal, W. R. Masking of alphabetic character recognition by dynamic visual noise. Perception \& Psychophysics, 1969, 6. 121-128.

Wheeler, D. D. Processes in word recognition. Cognitive Psychology, 1970, 1, 59-85.

\section{NOTE}

1. The program for the dynamic visual noise mask is due to Frank M. Goode 